\title{
A STUDY ON FORMAL DEDUCTIONS IN THE PRIMITIVE LOGIC
}

\author{
KATUZI ONO
}

To Kiyoshi Noshiro on the occasion of his 60 th birthday.

\section{Introduction.}

Main purpose of the present paper is to study formal deductions described along the line of my former work [1]. In the present paper, I restrict myself to the primitive logic ${ }^{1}$. To extend this method to other logics such as the lower classical predicate logic or the intuitionistic predicaste logic, my way of practical discription has to undergo a certain extent of modification.

The primitive logic can be formulated so as to admit a certain amount of round-about reasonings. The formulation $\boldsymbol{L} \boldsymbol{O}$ introduced in my former work gives an example of this kind. We can also formulate the same logic in such a manner that no round-about reasoning is admitted. The formulation [LO] introduced in (1) of the present paper is a system of such kind. Both systems $\boldsymbol{L} \boldsymbol{O}$ and $[\boldsymbol{L O}]$ are so formulated that reasonings in both systems can be described in my way of practical description.

Reasonings described practically in my way are called PROOF-NOTES. Main purpose of the present paper can be also stated as a meta-logical study of proof-notes, which would be duly called a kind of proof-theories.

In (2), I will describe a general aspect of proof-notes. I will study on some elementary transformations of proof-notes in the same section.

One of the most remarkable results in the proof theory must be Gentzen's cut-elimination theorem. Deductions in $[\boldsymbol{L O}]$ corresponds exactly to cut-free deductions in Gentzen's formalism. ${ }^{2}$ If we describe a proof-note in the primitive logic following our natural way of reasoning, the proof-note

Received July 21, 1966.

1) The primitive logic has been introduced in my work [2] under the name PRIMITIVE SYSTEM OF POSITIVE LOGIC. The name PRIMITIVE LOGIC has been introduced in my work [3].

2) See Gentzen [1]. 
would contain a certain amount of detours although it would be hard to find out where detours really occur.

In reality, detour-free reasoning is not always advisable. It makes deductions occasionally longer, occasionally even obscurer. For instance, even when we deal with a number of special cases of a general rule without having any concern about the general rule itself, our reasoning would occasionally become transparent by proving the general rule at first and then deduce its special cases. However, this way of reasoning could be hardly called detour-free. Sticking to detour-free reasoning, we have to go through a weary task of deducing the special cases of the same general rule repeatedly again and again.

We are interested in detour-free reasoning from the meta-logical point of view. It is really important to know that any reasoning with detours can be modulated into a reasoning without detours. Since we can interpret all the $\boldsymbol{J}$ - and $\boldsymbol{K}$-series logics faithfully in the primitive logic $\boldsymbol{L O}^{3}$, it would be enough for theoretical purposes to prove this with respect to the primitive logic only.

Inference rules of the logic [ $\boldsymbol{L O}$ ] differ from that of the logic $\boldsymbol{L O}$ only by that each proposition in inference rules of $[\boldsymbol{L O}]$ is given either of the two standings $\mathrm{B}$ (bare) or $\mathrm{C}$ (clad). If we disregard the standings of propositions of a proof-note in $[\boldsymbol{L O}]$, we would have a proof-note of $\boldsymbol{L O}$. However, we can not always transform every proof-note of $\boldsymbol{L O}$ into a right proof-note of $[\boldsymbol{L O}]$ simply by giving a standing to each proposition occurring in it. We can expect this only for detour-free proof-notes of $\boldsymbol{L O}$.

In (3), I would like to show a characteristic feature of the logic [LO] by proving some theorems such as the theorem: Any proof-note of a proposition $\mathfrak{F}$ in $[\boldsymbol{L O}$ ] without any redundant steps contains only sub-formulas of $\mathfrak{F}$.

In (4), I prove a theorem corresponding to the cut-elimination theorem in Gentzen's formalism. The theorem reads: Any proposition provable in $\boldsymbol{L O}$ is also provable in $[\boldsymbol{L O}]$.

3) See Ono [3] and [4]. I call the intuitionistic predicate logic, the minimal predicate logic, and the positive predicate logic altogether $\boldsymbol{J}$-SERIES LOGICS, and the logics obtained

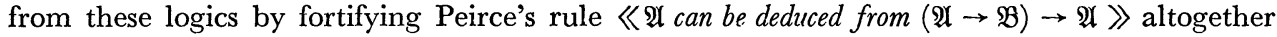
$\boldsymbol{K}$-SERIES LOGICS. In fact, the lower classical predicate logic can be regarded as the fortified intuitionistic predicate logic by Peirce's rule. 


\section{(1) The logics $\mathrm{LO}$ and [LO].}

The primitive logic $\boldsymbol{L O}$ is the logic having only two logical constants IMPLICATION $\rightarrow$ and UNIVERSAL QUANTIFICATION $(\quad)$ and having the following inference rules:

$\boldsymbol{F}$ : The step $\mathfrak{A}$ can be deduced from the step $\mathfrak{A}$.

$\boldsymbol{I}:$ The step $\mathfrak{B}$ can be deduced from the steps $\mathfrak{A}$ and $\mathfrak{U} \rightarrow \mathfrak{B}$.

$I^{*}:$ The step $\mathfrak{A} \rightarrow \mathfrak{B}$ can be deduced from the fact that $\mathfrak{B}$ is deducible from $\mathfrak{A}$.

$\boldsymbol{U}$ : The step $\mathfrak{A}(t)$ can be deduced from the step $(x) \mathfrak{A}(x)$ as far as $\mathfrak{A}(u)$ contains no free variable $x$ at all.

$U^{*}$ : The step $(x) \mathfrak{A}(x)$ can be deduced from the fact that $\mathfrak{A}(t)$ is deducible for any variable $t$ whatever $(\forall t !)$.

Next, the logic $[\boldsymbol{L O}]$. In proof-notes of this logic, we mark some propositions by enclosing them in pairs of brackets to show their standings. Any proposition in proof-notes of $[\boldsymbol{L O}]$ is called CLAD or BARE according as it is enclosed or is not enclosed in a pair of brackets.

The followings are the inference rules of the logic $[\boldsymbol{L O}]$ :

$[\boldsymbol{F}]$ The step $\mathfrak{A}$ can be deduced from the step $\mathfrak{A}$ as well as from the step $[\mathfrak{U}]$.

[I] The step [] can be deduced from the steps $\mathfrak{A}$ and $[\mathfrak{X} \rightarrow \mathfrak{B}]$.

$\left[\boldsymbol{I}^{*}\right] \quad$ The step $\mathfrak{A} \rightarrow \mathfrak{B}$ can be deduced from the fact that $\mathfrak{B}$ is deducible from [थ्U] .

[U] The step $[\mathfrak{U}(t)]$ can be deduced from the step $[(x) \mathfrak{A}(x)]$ as far as $\mathfrak{A}(u)$ contains no free variable $x$ at all.

[U⿱一⿻上丨 ${ }^{*}$ The step $(x) \mathfrak{U}(x)$ can be deduced from the fact that $\mathfrak{A}(t)$ is deducible for any variable $t$ whatever, i.e., from the fact that the step $\mathfrak{A}(t)$ is deducible from the step $\forall t$ !

Any proposition $\mathfrak{P}$ is called PROVABLE in $[\boldsymbol{L O}]$ if and only if the step $\mathfrak{F}$ is deducible in $[\boldsymbol{L O}]$. Needless to say, any proposition provable in $[\boldsymbol{L O}]$ is also provable in $\boldsymbol{L O}$.

\section{(2) Proof-notes.}

At first, a slightly improved index-word system of my formalism. Next, 
a few discussions concerning transformations of proof-notes into other proofnotes. Although I discuss these matters with respect to the primitive logic in this section, we can apply them to the $\boldsymbol{J}$ - and $\boldsymbol{K}$-series logics.

Every step in any proof-note is introduced and denoted by its introductory index. Any index-word is a sequence of letters including the null sequence. We usually make use of letters $\boldsymbol{A}, \boldsymbol{b}, \boldsymbol{c}, \ldots$ assuming the usual alphabetical order between them. Further, the natural numbers $0,1,2, \ldots$ are associated to these letters, and the letters are regarded as standing for these numbers. Namely, $\boldsymbol{A}$ denotes $0, \boldsymbol{b}+1$ denotes $\boldsymbol{c}, \boldsymbol{p}+(\boldsymbol{d}-\boldsymbol{b})$ denotes $\boldsymbol{r}$, and so on. We denote sequences of letters by underlined single letters. For example, $\underline{\boldsymbol{a}}, \underline{\boldsymbol{b}}, \underline{\boldsymbol{c}}, \ldots$ denote sequences of letters, and $\underline{\boldsymbol{o}}$ denotes the null sequence. If $\boldsymbol{b} \boldsymbol{b}$ is denoted by $\underline{\boldsymbol{s}}$ and $\boldsymbol{p q} \boldsymbol{A}$ is denotes by $\underline{\boldsymbol{t}}$, then $\underline{\boldsymbol{s}} \underline{\boldsymbol{t}}$ denotes naturaly $\boldsymbol{b} \boldsymbol{b} \boldsymbol{h} \boldsymbol{p q} \boldsymbol{A}$.

Ordering of steps arranged in the lexicographic order of their indexwords is called NATURAL ORDER of steps. In our real thinking, we reason mostly in this order. $\underline{s}_{N} \underline{t}$ means that the index-word $\underline{s}$ stands before the index-word in $\boldsymbol{t}$ the natural order. The notation $\underset{N}{\widehat{N}}$ would require no further explanation. Needless to say, the natural order is a linear order.

I will introduce here two other orderings of steps called FUNDAMENTAL ORDER and BASIS ORDER.

To illustrate the fundamental order of steps, I will take up an auxiliary letter $\diamond$ which has never been employed in expressing index-words. I will normalize the lengths of all the index-words of any proof-note by adding a sufficient number of $\diamond$ 's at the end of each index-word. Let us denote by $\boldsymbol{s} \diamond$ the normalized index-wiord thus obtained from the index-word $\underline{s}$. In the fundamental order, steps $\underline{s}$ are arranged in the lexicographic order of the normalized index-words $s \diamond$ regarding $\diamond$ as the last letter in the order of letters. It should be noticed that the order of index-words thus defined is independent of choice of length for normalization of index-words. Fundamental order is denoted by $\underset{F}{<}$ and $\underset{F}{\leq}$. It is also a linear order.

The basis order (notation: $<$ and $\leq$ ) is the minimal relation satisfying the conditions: $\underline{s} p<\underline{s} q \underline{t}$ for $p \underset{N}{ } q$, and $\underline{s} p<\underline{s}$. Any step $\underline{s}$ is qualified to be a basis of the step $\underline{t}$ if and only if $\underline{s}<\underline{t}$. Naturally, the basis order 
is a partial order. It should be remarked that the basis order is a sub-order of the fundamental order.

For practical description of proof-notes, it is convenient to employ two special letters $\boldsymbol{A}$ and $\in$ for index-words regarding them as the first and the last letter, respectively. Naturally, $\diamond$ is regarded as to stand after $\epsilon$ when we make use of the auxiliary letter $\diamond . \epsilon$ might be nicely interpreted as standing for a sufficiently large number.

If a step $\underline{\boldsymbol{s}}$ in a proof-note is deduced from the step $\underline{\boldsymbol{u}}$ by the inference rules $\boldsymbol{F}$ or $\boldsymbol{U}$, or if it is deduced from the steps $\underline{\boldsymbol{u}}$ and $\underline{\boldsymbol{v}}$ by the inference rule $\boldsymbol{I}$, the index-word $\underline{s}$ should never end with the letter $\boldsymbol{A}$ and the index-


over, there should never occur any step of the form $\boldsymbol{s} \boldsymbol{p} \boldsymbol{t}$ in the proof-note. The step $\underline{\boldsymbol{u}}$ or the steps $\underline{\boldsymbol{u}}$ and $\underline{\boldsymbol{v}}$ are called the REFERENCE STEPS of the step $\underline{\boldsymbol{s}}$ in the respective cases. If the step $\ll \underline{\boldsymbol{s}}) \mathfrak{A} \rightarrow \mathfrak{B} \gg$ is deduced by the inference rule $I^{*}$ from the fact that the step $\mathfrak{B}$ is deducible from the step $\mathfrak{A}$, the index-word $\underline{\boldsymbol{s}}$ should never end with the letter $\boldsymbol{A}$ and the introductory indices of the steps $\mathfrak{A}$ and $\mathfrak{B}$ should be $\boldsymbol{s} \boldsymbol{A}$ and $\underline{\boldsymbol{s}} \in$, respectively. Also, if the step $\ll \underline{s})(x) \mathfrak{A}(x) \gg$ is deduced by the inference rule $\boldsymbol{U}^{*}$ from the fact that the step $\mathfrak{A}(t)$ is deducible from the step $\forall t$ !, the index-word $\underline{s}$ should never end with the letter $\boldsymbol{A}$ and the introductoy indices of the steps $\forall t !$ and $\mathfrak{A}(t)$ should be $\underline{\boldsymbol{A}}$ and $\underline{\boldsymbol{s}} \in$, respectively.

For any denomination of the form $\ll \underline{s} \boldsymbol{A}) \forall t$ ! 》, the variable $t$ should not occur free in any step $\underline{\boldsymbol{u}}$ standing before $\underline{\boldsymbol{s}} \boldsymbol{A}$ in the basis order $(\underline{\boldsymbol{u}}<\underline{\boldsymbol{s}} \boldsymbol{A})$. The denomination is valid only for steps $\underline{\boldsymbol{w}}$ satisfying $\underline{\boldsymbol{s}} \boldsymbol{A} \leq \underline{\boldsymbol{w}}$ and $\underline{\boldsymbol{s}} \boldsymbol{A} \underset{N}{\underline{\boldsymbol{w}}}$.

Now, let $\Pi$ be any proof-note. For any steps $\underline{s}$ of $\Pi$, let us denote by $\Pi[-\boldsymbol{s}]$ and $\Pi[\underline{s}-]$ the first part of $\Pi$ up to the step $\underline{s}$ and the last part of $\Pi$ from the step $\underline{s}$ in the fundamental order of steps, respectively. Any step of the form $\underline{\boldsymbol{r}} \boldsymbol{A}$ satisfying simultaneously $\underline{\boldsymbol{r}} \boldsymbol{A} \leq \underline{\boldsymbol{s}}$ and $\underline{\boldsymbol{r}} \boldsymbol{A} \leq \underline{\boldsymbol{s}}$ is called an ASSUMPTION STEP of $\boldsymbol{s}$. The set of steps of the form $\underline{s} \boldsymbol{p}$ is called the FRAME-WORK of $\underline{s}$. Any step having non-empty framework is called a LEMMA. The step $\underline{s} \boldsymbol{A}$ and $\underline{s} \in$ are called the REFERENCE STEPS of the lemma step $\underline{s}$. Any step $\underline{\boldsymbol{t}}$ of $\Pi[-\boldsymbol{s}]$ which is referred to by no step in $\Pi[-\boldsymbol{s}]$ is called a TERMINAL STEP of $\Pi[-\underline{s}]$.

If a series $\Pi$ of steps has the characteristic properties of proof-notes above mentioned, then $\Pi[-\underline{s}]$ also has the same properties. Any series $\Gamma$ 
of steps having these properties is called a SEMI-PROOF-NOTE. For a complete proof-note, we expect further that for any step of the form $\underline{s} \underline{t}$ there is a lemma step $\underline{\boldsymbol{s}}$ whose frame-work begins with $\underline{\boldsymbol{s}} \boldsymbol{A}$ and ends with $\underline{\boldsymbol{s}} \in$. Accordingly, any proof-note $\Pi$ can be regarded as beginning with the step $\boldsymbol{A}$ and ending with the step $\underline{\boldsymbol{o}}$ (null sequence index-word). Hence, $\Pi[\boldsymbol{A}-]$ as well as $\Pi[-\underline{0})$ is the proof-note $\Pi$ itself. $\Pi$ can be regarded as a proof-note of the proposition of the last step $\underline{o}$ of $\Pi$. Evidently, any proofnote is also a semi-proof-note.

The step $\underline{\boldsymbol{o}}$ is surely a terminal step of $\Pi$. However, $\Pi[-\boldsymbol{o}]$ may have some terminal steps other than o. In this case, these terminal steps are called REDUNDANT: Any proof-note containing no redundant terminal steps is called WASTELESS.

THeORem 1. Any proposition provable in LO (or in [LO]) can be proved by a wasteless proof-note in $\mathbf{L O}$ (or in $[\boldsymbol{L O}]$ ).

Proof. Let $\Pi$ be any proof-note of a proposition $\mathfrak{P}$ containing a redundant terminal steps $\underline{\boldsymbol{s}}$ which is a step of the form $\boldsymbol{t} \boldsymbol{p}$. Then, $\boldsymbol{p}$ is neither $\boldsymbol{A}$ nor $\in$, because $\underline{\boldsymbol{t}} \boldsymbol{A}$ as well as $\underline{\boldsymbol{t}} \in$ is a reference step of the step $\underline{\boldsymbol{t}}$. Now, any step of the form $\underline{s u}$ is a reference step of no step other than those steps of the same form. Accordingly, if we delete all the steps of the form $\boldsymbol{s u}$, we have a proof-note of $\mathfrak{P}$ containing a smaller number of redundant terminal steps. Hence, after a finite number of steps of the same process, we would finally arrive at a wasteless proof-note of the proposition $\mathfrak{F}$.

Remark. In practice, no letters are skipped over in making index-words. Accordingly, if there occur index-words $\underline{s} b$ and $\underline{s} \boldsymbol{e}$, for example, we can well expect occurrence of steps $\underline{s} \boldsymbol{c}$ and $\underline{s} \boldsymbol{d}$ in the same proof-note. However, this is not required formally. We can easily repair any skipping indexword system such as the system just obtained in the proof of the preceding theorem into a non-skipping index-word system.

Any semi-proof-note $\Delta$ is called a MODULATION of another semiproof-note $\Gamma$ by $\Theta$ if and only if $\Theta$ is one-to-one correspondence $\underline{s} \leftrightarrow \Theta(\underline{s})$ between the whole set of index-words of $\Gamma$ and a set of index-words of $\Delta$ such that

1) The proposition or denomination of any step $\underline{s}$ in $\Gamma$ is the same as that of the step $\Theta(\underline{s})$ in $\Delta$. 
2) Any step $\underline{v}$ in $\Delta$ is an assumption step of $\Theta(\underline{s})$ if and only if there is an assumption step $\underline{\boldsymbol{u}}$ of $\underline{\boldsymbol{s}}$ in $\Gamma$ whose $\Theta$-image is $\underline{\boldsymbol{v}}(\underline{\boldsymbol{v}}=\Theta(\underline{\boldsymbol{u}}))$.

3) $\underline{\boldsymbol{s}}_{N} \underline{\boldsymbol{t}}$ and $\underline{\boldsymbol{s}}_{F} \underline{\boldsymbol{t}}$ imply $\Theta(\underline{\boldsymbol{s}}) \underset{N}{<} \Theta(\underline{\boldsymbol{t}})$ and $\Theta(\underline{\boldsymbol{s}}) \underset{F}{<} \Theta(\underline{\boldsymbol{t}})$, respectively. If a semi-proof-note is a modulation of $\Gamma$ by $\Theta$, it is denoted by $\Theta(\Gamma)$.

Theorem 2. Any modulation of a modulation of a semi-proof-note is a modulation of the semi-proof-note.

TheOrem 3. For any modulation of a semi-proof-note by $\Theta$, the lengths of the index-words $\underline{s}$ and $\Theta(\underline{s})$ are necessarily equal.

By virtue of Theorem 3, for any modulation $\Theta(\Gamma)$ each letter in any index-word $\underline{s}$ of $\Gamma$ corresponds to a letter of $\Theta$-image $\Theta(\underline{s})$. The corresponding letter of $x$ in a index-word $\underline{s}$ is called the $\Theta$-IMAGE of $x$ in $\underline{s}$. If the $\Theta$-image of every $\epsilon$ in any index-word $\underline{s}$ of a semi-proof-note $\Gamma$ is necessarily $\epsilon$, the modulation $\Theta(\Gamma)$ is called NORMAL.

Theorem 4. Let $\Gamma$ be a proof-note and $\Theta(\Gamma[-\underline{s}])$ be normal. Then, $\Theta(\Gamma[-\underline{s}])$ can be extended to a modulation $\Theta^{*}(\Gamma)$ of the whole proof-note $\Gamma$, for which $\Theta^{*}(\Gamma)\left[\Theta^{*}(\underline{s})\right.$ - $]$ has the same number of steps as $\Gamma[\underline{s}-]$.

Proof. For any step $\underline{\boldsymbol{u}}$ standing before $\underline{\boldsymbol{s}}$ in the fundamental order $(\underline{\boldsymbol{u}} \leq \underline{\boldsymbol{s}})$, define $\Theta^{*}(\underline{\boldsymbol{u}})$ by $\Theta(\underline{\boldsymbol{u}})$. Any step $\underline{\boldsymbol{v}}$ standing after $\underline{\boldsymbol{s}}$ in the fundamental order $(\underline{\boldsymbol{s}} \underset{F}{\boldsymbol{v}})$ can be denoted in the form $\underline{\boldsymbol{r}} \boldsymbol{q} \underline{\boldsymbol{w}}$ where $\underline{\boldsymbol{s}}$ can be denoted in the form $\boldsymbol{r} \boldsymbol{p} \underline{z}$ for $\boldsymbol{p}$ standing before $\boldsymbol{q}$ in the order of letters. Now, let $\underline{\boldsymbol{r}}^{*} \boldsymbol{p}^{*} \underline{\boldsymbol{z}}^{*}$ be the $\Theta$-image of $\underline{\boldsymbol{r} \boldsymbol{p}} \boldsymbol{z}$. Then, we define $\Theta^{*}(\underline{\boldsymbol{v}})$, i.e. $\Theta^{*}(\underline{\boldsymbol{r} q} \underline{\boldsymbol{w}})$, by $\underline{\boldsymbol{r}}^{*}\left(\boldsymbol{p}^{*}+(\boldsymbol{q}-\boldsymbol{p})\right) \underline{\boldsymbol{w}}$. If $\boldsymbol{p}$ is $\in$, then $\Theta^{*}(\underline{\boldsymbol{v}})$ is defined by $\underline{\boldsymbol{r}}^{*} \in \underline{\boldsymbol{w}}$. The reference indices of any step after the step $\underline{s}^{*}$ in $\Theta^{*}(\Gamma)$ are the $\Theta^{*}$ images of the reference indices of the original step. We can see easily that $\Theta^{*}(\Gamma)$ is a modulaion of the whole proof-note $\Gamma$.

Thoerem 5. Any modulation of a proof-note of a proposition is a proof-note of the same proposition.

Proof. Let $\Pi$ be any proof-note of $\mathfrak{B}$ and $\Theta(\Pi)$ be a modulation of $\Pi$. Then, $\mathfrak{F}$ is the proposition of $\Theta(\underline{\boldsymbol{o}})$ in $\Theta(\Pi)$, and $\Theta(\underline{\boldsymbol{o}})$ is the last step of $\Theta(\Pi)$ because $\Theta(\underline{o})$ is necessarily a null-sequence index-word according to Theorem 3 . 


\section{(3) A characteristic feature of proof-notes of $[\mathrm{LO}]$.}

The following theorems seem to show a characteristic feature of proofnotes of $[\boldsymbol{L O}]$.

THEOREM 6. For every step $\underline{\boldsymbol{s}}$ in any semi-proof-note of $[\boldsymbol{L O}]$ hold the following conditions:

1) If the formula of any step $\underline{\boldsymbol{s}}$ is clad, it is a sub-formula of a clad proposition in the reference steps of $\underline{s}$.

2) Any bare proposition in the reference steps of $\underline{\boldsymbol{s}}$ is either a sub-formula of the proposition of $\underline{\boldsymbol{s}}$ (in this case, the proposition should be bare) or a sub-formula of a clad proposition in the reference steps of $\underline{\boldsymbol{s}}^{\mathbf{4})}$.

TheORem 7. Any proposition occurring in any wasteless proof-note of a proposition $\mathfrak{B}$ is a sub-formula of the proposition $\mathfrak{\Re}$.

This theorem can be regarded as a special case of the following more extensive theorem.

THEOREM 8. Let $\Pi$ be any proof-note in the logic [LO] and let $\underline{\boldsymbol{s}}$ be any step of $\Pi$. Then,

$\boldsymbol{A}(\boldsymbol{s})$ : Any clad formula of the step $\underline{\boldsymbol{s}}$ is a sub-formula of the proposition of an assumption step of $\underline{\boldsymbol{s}}$.

$\boldsymbol{B}(\boldsymbol{s})$ : Any formula in $\Pi[-\boldsymbol{s}]$ is either a sub-formula of a bare formula in terminal steps of $\Pi[-\boldsymbol{s}]$ or a sub-formula of a proposition in assumption steps of $\underline{\boldsymbol{s}}$.

Proof. We can easily see that $\boldsymbol{A}(\underline{\boldsymbol{s}})$ as well as $\boldsymbol{B}(\underline{\boldsymbol{s}})$ holds true for the first step $\underline{s}$ in any proof-note $\Pi$ arranged in the fundamental order, since the first step of any proof-note must be an assumption step of itself. So, by assuming $\boldsymbol{A}(\underline{\boldsymbol{t}})$ and $\boldsymbol{B}(\underline{\boldsymbol{t}})$ for every step $\underline{\boldsymbol{t}}$ of $\Pi$ standing before $\underline{s}$ in the fundamental order $\left(\underline{\boldsymbol{t}}_{F} \boldsymbol{s}\right)$, I will show $\boldsymbol{A}(\underline{\boldsymbol{s}})$ and $\boldsymbol{B}(\boldsymbol{s})$. Let us denote the step standing just before $\underline{s}$ by $\underline{r}$.

The step $\underline{s}$ is either an assumption step of itself (Case 1), or it is deduced from a step $\underline{\boldsymbol{u}}$ or from steps $\underline{\boldsymbol{u}}$ and $\underline{\boldsymbol{v}}$ (Case 2), or it is deduced from the fact that the step $\underline{\boldsymbol{s}} \in$ is deducible from the step $\underline{\boldsymbol{s}} \boldsymbol{A}$ (Gase 3). of $\mathfrak{B}$.

4) Here $I$ call $[\mathfrak{X}]$ as well as $\mathfrak{A}$ a sub-formula of $[\mathfrak{B}]$ as well as of $\mathfrak{B}$ if $\mathfrak{A}$ is a sub-formula 
Case 1, where the step $\underline{\boldsymbol{s}}$ is an assumption step of itself: $\boldsymbol{A}(\underline{\boldsymbol{s}})$ as well as $\boldsymbol{B}(\underline{s})$ holds true evidently.

Case 2, where the step $\underline{\boldsymbol{s}}$ is deduced from a step $\underline{\boldsymbol{u}}$ or from steps $\underline{\boldsymbol{u}}$ and $\underline{\boldsymbol{v}}$ : Any assumption step of $\underline{\boldsymbol{r}}$ as well as any assumption step of $\underline{\boldsymbol{u}}$ as well as any assumption step of $\underline{\boldsymbol{v}}$ is also an assumption step of $\underline{\boldsymbol{s}}$, and any terminal step of $\Pi[-\underline{r}]$ other than $\underline{\boldsymbol{u}}$ and $\underline{\boldsymbol{v}}$ is also a terminal step of $\Pi[\boldsymbol{-} \underline{\boldsymbol{s}}]$.

If the proposition of the step $\underline{s}$ is clad, it must be a sub-formula of a clad formula $[\boldsymbol{A}]$ of the steps $\underline{\boldsymbol{u}}$ or $\underline{\boldsymbol{v}}$ according to Theorem 6 , so the formula $\boldsymbol{A}$ must be a sub-formula of the proposition $\mathfrak{B}$ of an assumption step $\underline{\boldsymbol{w}}$ of $\underline{\boldsymbol{u}}$ or $\underline{\boldsymbol{v}}$ by assumption. Since $\underline{\boldsymbol{w}}$ is also an assumption step of $\underline{\boldsymbol{s}}, \boldsymbol{A}(\underline{\boldsymbol{s}})$ holds.

Next, any proposition $\mathfrak{A}$ in $\Pi[-\boldsymbol{s}]$ must be either the proposition of the step $\underline{s}$ (Case 2. 1) or a proposition in steps of $\Pi[-\underline{r}]$ (Case 2. 2).

Case 2. 1, where $\mathfrak{A}$ is the propostiion of the step $\underline{s}$ : If $\mathfrak{A}$ is bare, $\mathfrak{A}$ is a sub-formula of the bare proposition $\mathfrak{A}$ of the terminal step $\boldsymbol{s}$ of $\Pi[-\boldsymbol{s}]$. If $\mathfrak{A}$ is clad, $\mathfrak{A}$ is a sub-formula of a proposition in assumption steps of $\underline{\boldsymbol{s}}$, because $\boldsymbol{A}(\underline{\boldsymbol{s}})$ holds true.

Case 2. 2, where $\mathfrak{A}$ is a proposition in steps of $\Pi[-\underline{r}]$ : By assumption, $\mathfrak{A}$ must be either a sub-formula of a bare proposition in terminal steps of $\Pi[-\boldsymbol{r}$ ] (Case 2. 2. 1) or a sub-formula of a proposition in assumption steps of $\underline{r}$ (Case 2. 2. 2).

Case 2. 2. 1, where $\mathfrak{A}$ is a sub-formula of the proposition $\mathfrak{B}$ of a terminal step $\underline{\boldsymbol{t}}$ of $\Pi[-\underline{\boldsymbol{r}}], \mathfrak{B}$ being bare: If $\underline{\boldsymbol{t}}$ coincides neither with $\underline{\boldsymbol{u}}$ nor with $\underline{\boldsymbol{v}}$, the step $\underline{t}$ is also a terminal step of $\Pi[-\boldsymbol{s}]$, so $\mathfrak{A}$ is a sub-formula of the bare proposition $\mathfrak{B}$ of the terminal step $\underline{t}$ of $\mathrm{II}[-\underline{s}]$. If $\underline{t}$ coincides with any one of $\underline{\boldsymbol{u}}$ or $\underline{\boldsymbol{v}}$, the bare proposition $\mathfrak{B}$ of the reference step $\underline{\boldsymbol{t}}$ of $\underline{\boldsymbol{s}}$ is a sub-formula of the proposition $\widetilde{C}$ of the step $\underline{s}$ which is bare, or a subformula of a clad proposition $\mathfrak{D}$ of reference steps $\underline{\boldsymbol{u}}$ or $\underline{\boldsymbol{v}}$ of $\underline{\boldsymbol{s}}$ by virtue of Theorem 6. In the former case, $\mathfrak{A}$ is surely a sub-formula of the bare proposition $\mathfrak{C}$ of the step $\underline{s}$. In the latter case, the clad proposition $\mathfrak{D}$ must be a sub-formula of the proposition of an assumption step $\underline{\boldsymbol{w}}$ of $\underline{\boldsymbol{u}}$ or $\underline{\boldsymbol{v}}$ by assumption. Since the assumption step $\underline{\boldsymbol{w}}$ of $\underline{\boldsymbol{u}}$ as well as of $\underline{\boldsymbol{v}}$ is also an assumption step of $\underline{\boldsymbol{s}}$, the proposition $\mathfrak{A}$ is a sub-formula of the proposition $D$ of the assumption step $\underline{\boldsymbol{w}}$ of $\underline{\boldsymbol{s}}$. 
Case 2. 2. 2, where $\mathfrak{A}$ is a sub-formula of the proposition of an assumption step $\underline{\boldsymbol{w}}$ of $\underline{\boldsymbol{r}}$ : Since the assumption step $\underline{\boldsymbol{w}}$ of $\underline{\boldsymbol{r}}$ is also an assumption step of $\underline{s}$, the proposition $\mathfrak{A}$ is a sub-formula of the proposition of the assumption step $\underline{w}$ of $\underline{s}$.

Hence, $\boldsymbol{B}(\underline{s})$ holds true anyway.

Case 3, where the step $\underline{s}$ is deduced from the fact that the step $\underline{s} \in$ is deducible from the step $\boldsymbol{s} \boldsymbol{A}$ : The proposition of the step $\underline{\boldsymbol{s}}$ is bare, so we have only to examine $\boldsymbol{B}(\underline{s})$. Any formula $\mathfrak{A}$ in $\Pi[-\underline{s}]$ is either the proposition of the step $\underline{s}$ (Case 3. 1) or a proposition in steps of $\Pi[-\underline{r}]$ (Case 3. 2).

Case 3. 1, where $\mathfrak{A}$ is the proposition of the step $\underline{s}$ : Since the proposition of the step $\underline{s}$ is bare, $\boldsymbol{B}(\underline{s})$ holds true trivially.

Case 3. 2, where $\mathfrak{A}$ is a proposition in the step of $\Pi[-\boldsymbol{r}]$ : By assumption, $\mathfrak{A}$ must be either a sub-formula of a proposition in terminal steps of $\Pi[-\underline{r}]$ (Case 3. 2. 1) or a sub-formula of a proposition in assumption steps of $\underline{r}$ (Case 3. 2. 2).

Case 3. 2. 1, where $\mathfrak{A}$ is a sub-formula of the proposition $\mathfrak{B}$ of a terminal step $\underline{\boldsymbol{t}}$ of $\Pi[-\underline{\boldsymbol{r}}]$, the proposition $\mathfrak{B}$ being bare: The step $\boldsymbol{t}$ can not coincide with $\underline{\boldsymbol{s}} \boldsymbol{A}$, because the proposition of $\boldsymbol{s} \boldsymbol{A}$, if any, is clad. Hence, either $\underline{t}$ is a terminal step of $\Pi[-\boldsymbol{s}]$ or $\underline{\boldsymbol{t}}$ coincides with $\underline{\boldsymbol{s}} \in$ whose proposition is a sub-formula of the bare proposition of the step $\underline{s}$. So, $\mathfrak{A}$ is a subformula of a bare proposition in terminal steps of $\underline{s}$ anyway.

Case 3. 2. 2, where $\mathfrak{A}$ is a sub-formula of the proposition $\mathfrak{B}$ of an assumption step $\underline{\boldsymbol{w}}$ of $\underline{\boldsymbol{r}}$ : Either the step $\underline{\boldsymbol{w}}$ is an assumption step of $\underline{\boldsymbol{s}}$ or it coincides with $\boldsymbol{s} \boldsymbol{A}$ whose proposition, if any, is a sub-formula of the bare proposition of the step $\boldsymbol{s}$. So, $\mathfrak{A}$ is either a sub-formula of the proposition of the assumption step $\underline{\boldsymbol{w}}$ of $\underline{\boldsymbol{s}}$, or a sub-formula of the bare proposition of the step $\underline{s}$.

Hence, $\boldsymbol{B}(\underline{s})$ holds true anyway.

\section{(4) Detour-free proof-notes in the primitive logic.}

Proof-notes in the logic $[\boldsymbol{L O}]$ can be regarded as detour-free. Any proposition provable in $[\boldsymbol{L O}]$ is evidently provable in $\boldsymbol{L O}$. Main purpose of this section is to prove that any proof-note in $\boldsymbol{L O}$ can be modulated into a detour-free proof-note in $[\boldsymbol{L O}]$. 
TheORem 9. Any proposition provable in the logic $\boldsymbol{L O}$ is also provable in the logic $[\boldsymbol{L O}]$.

Proof. Let $\mathfrak{F}$ be any proposition provable in $\boldsymbol{L} \boldsymbol{O}$ and let $\Pi$ be any proof-note of $\mathfrak{F}$ in $\boldsymbol{L O}$ arranged in the fundamental order of steps. I will show that II can be modulated into a right proof-note of the same proposition $\mathfrak{F}$ in $[\boldsymbol{L O}]$.

The modulation is carried out step by step. If $\Pi$ can not be modulated into a right proof-note of $[\boldsymbol{L O}]$ simply by enclosing some propositions in pairs of brackets, I modulate a semi-proof-note of the form $\Pi[-\boldsymbol{s}]$ into a semiproof-note $\Pi *\left[-\underline{s}^{*}\right]$ which is easier to be modulated into a semi-proof-note of $[\boldsymbol{L O}]$. By virtue of Theorems 2, 4, and 5, we can thus obtain a new proof-note of the same proposition $\mathfrak{B}$ which is easier to be modulated into a right proof-note of $\mathfrak{P}$ in $[\boldsymbol{L O}]$.

Namely, let $\Gamma$ be any proof-note in $\boldsymbol{L} \boldsymbol{O}$ and $\underline{s}$ be any step of it. To make $\Gamma[\boldsymbol{-} \boldsymbol{s}]$ a right semi-proof-note in $[\boldsymbol{L O}]$, each step $\underline{\boldsymbol{t}}$ of $\Gamma[\boldsymbol{-} \boldsymbol{s}]$ should have the standing of bare $(B)$ or clad $(C)$ according to two requests, unless $\boldsymbol{t}$ is a denomination. The first request is that such a standing should be given to the step $\underline{\boldsymbol{t}}$ so as to be deducible in $[\boldsymbol{L O}]$ if it is a deduced step originally. The second request is that such a standing should be given to the step $\underline{t}$ so as to be qualified as a logical basis in $[\boldsymbol{L O}]$ for steps in $\Gamma[\boldsymbol{-} \boldsymbol{s}]$ referring to it originally. These two requests occasionally contradict.

For any step $\underline{t}$, a single definite standing is requested by the first request, but occasionally the opposite standing or occasionally even the both standings are requested by the second request. If the standing $\mathrm{B}$ (or $\mathrm{C}$ ) is requested by the first request and the opposite standing $\mathrm{C}$ (or $\mathrm{B}$ ) or the both standings $\mathrm{B}$ and $\mathrm{C}$ are requested by the second request for a step, the step is called B-STEP (C-STEP). If we modulate the proof-note $\Pi$ into such a proof-note $\Pi^{*}$ where every step is neither B-step nor C-step, we can modulate $I I$ into a proof-note of $[\boldsymbol{L O}]$. Naturally, the inference rule $[\boldsymbol{F}]$ can be taken so as to avoid occurrence of B- or C-steps as possible.

Any proof-note with some C-steps can be modulated into a proof-note without C-steps. This can be proved by showing that any proof-note with some C-steps can be modulated into a proof-note with a smaller number of C-steps. Namely, let $\langle\underline{t} \boldsymbol{h}) \mathfrak{B} / \ldots \gg$ be any C-step in a proof-note $\Gamma$. Then, I will define $\Theta$-image of any step of the form $\underline{t} \boldsymbol{k} \underline{w}$ for any letter $\boldsymbol{k}$ 
standing after $\boldsymbol{h}$ by that $\Theta(\underline{\boldsymbol{t}} \boldsymbol{k} \underline{\boldsymbol{w}})$ is $\underline{\boldsymbol{t}}(\boldsymbol{k}+1) \underline{\boldsymbol{w}}$ and $\Theta$-image of any step $\underline{\boldsymbol{u}}$ of other forms by that $\Theta(\underline{\boldsymbol{u}})$ is $\underline{\boldsymbol{u}}$ itself. Also, a new step $\ll \underline{\boldsymbol{t}}(\boldsymbol{h}+1)) \mathfrak{B} / \underline{\boldsymbol{t}} \boldsymbol{h} 》$ is added in the modulation $\Theta(\Gamma)$. Any reference index $\underline{\boldsymbol{u}}$ other than $\boldsymbol{t} \boldsymbol{h}$ is replaced by $\Theta(\underline{\boldsymbol{u}})$ in the modulation $\Theta(\Gamma)$. Any reference index $\boldsymbol{t} \boldsymbol{h}$ of a step $\underline{\boldsymbol{w}}$ remains to be $\underline{\boldsymbol{t}} \boldsymbol{h}$ if $\underline{\boldsymbol{w}}$ is deduced either by the inference rule $\boldsymbol{I}$ for the proposition $\mathfrak{P}$ being $\mathfrak{A} \rightarrow \mathfrak{B}$ of $\boldsymbol{I}$, or by the inference rule $\boldsymbol{I}^{*}$ for the proposition $\mathfrak{B}$ being $\mathfrak{A}$ of $\boldsymbol{I}^{*}$, or by the inference rule $\boldsymbol{U}$ for the proposition $\mathfrak{B}$ being $(x) \mathfrak{A}(x)$ of $\boldsymbol{U}$. Any other reference indices $\underline{\boldsymbol{t}} \boldsymbol{h}$ should be replaced by $\underline{\boldsymbol{t}}(\boldsymbol{h}+1)$. Thus, we can obtain a modulation $\Theta(\Gamma)$ having a smaller number of $\mathrm{C}$-steps than $\Gamma$.

It should be noticed that the number of B-steps of the modulation $\Theta(\Gamma)$ is not greater than that of the original proof-note $\Gamma$.

Now, I would like to show that also B-steps are removable from semiproof-notes. Because the proposition of any B-step must be a proposition of the form $\mathfrak{B} \rightarrow \mathfrak{D}$ or $(x) \mathfrak{B}(x)$, there is no B-step having no logical constants. Hence, removability of B-steps could be proved by complete induction. Namely, I will call the maximum number of logical constants in B-steps of a semi-proof-note $\Gamma$ by $R A N K$ of $\Gamma(\operatorname{Rank}(\Gamma))$ and I will prove removability of B-steps by complete induction with respect to ranks of semi-proof-notes. If $\Gamma$ has no B-step, I will put $\operatorname{Rank}(\Gamma)=0$.

I will prove that, for any semi-proof-note without C-steps, there is a normal modulation of it with no B-steps and no C-steps, assuming the same thing for every semi-proof-note of lower ranks. Namely, let $\Gamma$ be any semiproof-note and let us assume that any semi-proof-note of lower ranks than $\Gamma$ without $\mathrm{C}$-steps has a normal modulation with no B-steps and no C-steps.

Now, let $\underline{s}$ be the first step in $\Gamma$ which makes $\operatorname{Rank}(\Gamma[-\underline{s}])$ positive. If $\operatorname{Rank}(\Gamma[-\boldsymbol{s}])<\operatorname{Rank}(\Gamma)$, there is a normal modulation of $\Gamma[-\boldsymbol{s}]$ with no B-steps and no C-steps. By virtue of Theorem 4, the modulation can be extended to a modulation $\Theta(\Gamma)$ of the whole proof-note with the same number of steps in $\Theta(\Gamma[\Theta(\underline{s})-])$ as in $\Gamma[\underline{s}-]$. Hence, if we take the first step $\underline{t}$ in $\Theta(\Gamma)$ which makes $\operatorname{Rank}(\Theta(\Gamma)[-\underline{t}])$ positive, $\Theta(\Gamma)[\underline{t}-]$ has a smaller number of steps than $\Gamma[\underline{s}-]$. Evidently, $\operatorname{Rank}(\Theta(\Gamma)) \leq \operatorname{Rank}(\Gamma)$ and $\Theta(\Gamma)$ has no $\mathrm{C}$-steps.

If $\operatorname{Rank}(\Gamma[-\underline{s}])=\operatorname{Rank}(\Gamma)$, the step $\underline{s}$ must be either deduced from steps of the forms $\ll \underline{p}) \mathfrak{P} \rightarrow \mathfrak{\downarrow}$ and $\langle\underline{\boldsymbol{q}}) \mathfrak{P} / \ldots \gg$ by the inference rule $\boldsymbol{I}$ or deduced from a step of the form $\ll \underline{p})(x) \mathfrak{P}(x) \gg$ by the inference rule 
$\boldsymbol{U}$. The lemma step $\boldsymbol{p}$ is the only B-step of $\Gamma[-\boldsymbol{s}]$ in both cases. The number of logical constants in the proposition of the lemma step $\boldsymbol{p}$ is equal to $\operatorname{Rank}(\Gamma)$.

Case 1, where the step $\ll \underline{\boldsymbol{s}}) \mathfrak{D} / \boldsymbol{p}, \boldsymbol{q} \gg$ is deduced from the steps $\underline{\boldsymbol{p}}$ and $\underline{\boldsymbol{q}}$ by the inference rule $\boldsymbol{I}$ : In this case $\underline{\boldsymbol{s}}$ is the only step in $\Gamma[-\boldsymbol{s}]$ whose proposition is $\precsim$ and which is deduced from the step $\boldsymbol{p}$ by the inference rule $\boldsymbol{I}$. The lemma step $\underline{\boldsymbol{p}}$ must have its frame-work $\ll \underline{\boldsymbol{p} A}) \mathfrak{P} \gg, \ldots$, $\ll \underline{p} \in) \bigcap / \ldots \gg$, say $k$ steps. Now, let us assume that $\underline{s}$ is an index-word of the form $\underline{\boldsymbol{r}} \boldsymbol{h}$ and $\underline{\boldsymbol{r} g}$ is the last index-word of the form $\underline{\boldsymbol{r}} \boldsymbol{x}$ standing before $\boldsymbol{s}$. To give a modulation $\Theta(\Gamma[-\boldsymbol{s}])$, we do not change any step of $\Gamma[-\underline{s}]$ except for the last step $\underline{s}$, even the reference indices remain unchanged. Corresponding to every step of the form $\underline{\boldsymbol{p} x \boldsymbol{w}}$ except for $\boldsymbol{x}$ being $\boldsymbol{A}$ or $\epsilon$, we make a new step $\boldsymbol{r}(\boldsymbol{g}+\boldsymbol{x}) \underline{w}$ having the same proposition or denomination as $\underline{\boldsymbol{p} x} \underline{\boldsymbol{w}}$, and corresponding to every step of the form $\underline{\boldsymbol{p}} \in \underline{\boldsymbol{w}}$, we make a new step $\underline{\boldsymbol{r}}(\boldsymbol{g}+\boldsymbol{k}) \underline{\boldsymbol{w}}(\underline{\boldsymbol{r}} \in \underline{\boldsymbol{w}}$ if $\boldsymbol{h}$ is $\epsilon)$ having the same proposition or denomination as $\underline{p} \in \underline{w}$. The $\Theta$-image of $\underline{\boldsymbol{s}}$ is $\underline{\boldsymbol{r}}(\boldsymbol{g}+\boldsymbol{k})(\underline{\boldsymbol{r}} \in$ if $\boldsymbol{h}$ is $\epsilon)$. In the new steps, the reference index $\underline{\boldsymbol{p} A}$ should be replaced by $\underline{\boldsymbol{q}}$, the reference indices of the form $\underline{p} \boldsymbol{w}$ other than $\underline{\boldsymbol{p}} \boldsymbol{A}$ should be replaced by its corresponding index-words of the form $\underline{\boldsymbol{r} z \underline{w}}$, and only in the last step $\underline{\boldsymbol{s}}$, the reference index $\underline{q}$ should be deleted before the replacement. We can easily see that a normal modulation of $\Gamma[-\underline{s}]$ without $\mathrm{C}$-steps is given by this correspondence. Only the step $\underline{\boldsymbol{q}}$ in the modulation $\Theta(\Gamma[-\underline{\boldsymbol{s}}])$ might possibly be a B-step, but it has a smaller number of logical constants than the original B-step $\underline{p}$. Hence, there is a normal modulation of $\Gamma[-\underline{s}]$ with no B-steps and no $\mathrm{C}$-steps by virtue of our assumption. According to Theorem 4, we can extend this modulation to a modulation $\Theta^{*}(\Gamma)$ of the whole proof-note in such way that $\Theta^{*}(\Gamma)\left[\Theta^{*}(\underline{s})\right.$ - $]$ has the same number of steps as $\Gamma[\underline{s}-]$. Consequently, if we take the first step $\underline{t}$ in $\Theta^{*}(\Gamma)$ which makes $\operatorname{Rank}\left(\Theta^{*}(\Gamma)[-\underline{t}]\right)$ positive, the number of steps of $\Theta^{*}(\Gamma)[\boldsymbol{t}-]$ is less than that of $\Gamma[\underline{s}-]$.

Case 2, where the step $\ll \underline{\boldsymbol{s}}) \mathfrak{P}(t) \mid \underline{\boldsymbol{p}} \gg$ is deduced from the step $\ll \underline{\boldsymbol{p}})(x) \mathfrak{P}(x) 》$ by the inference rule $\boldsymbol{U}$ : In this case, $\underline{\boldsymbol{s}}$ is the only step in $\Gamma[-\boldsymbol{s}]$ which is deduced from the step $\underline{p}$ by the inference rule $\boldsymbol{U}$. The lemma step $\underline{\boldsymbol{p}}$ must have its frame-work of the form $\ll \underline{p} \boldsymbol{A}) \forall u$ ! 》, . , $\langle\underline{\boldsymbol{p}} \in) \mathfrak{P}(u) \ldots \gg$. The way of adding new steps is almost the same as that of Case 1 . We 
have only to remark that the free variable $u$ in the newly added steps should be replaced by the variable $t$. We can see that the correspondence gives a normal modulation of $\Gamma[-\boldsymbol{s}]$ with no B-steps and no C-steps by virtue of our assumption for denominating quantification. According to Theorem 4, we can extend this modulation to a modulation $\Theta(\Gamma)$ of the whole proof-note in such way that $\Theta(\Gamma)[\Theta(\underline{s})$ - ] has the same number of steps as $\Gamma[\underline{s}-]$. Consequently, if we take the first step $\underline{t}$ in $\Theta(\Gamma)$ which makes $\operatorname{Rank}(\Theta(\Gamma)[-\underline{t}])$ positive, the number of steps of $\Theta(\Gamma)[\underline{t}-]$ is less than that of $\Gamma[\underline{s}-]$.

Thus we have proved in all cases that for any proof-note $\Gamma$ without C-steps, we can find out a modulation $\Delta$ without C-steps such that: Let $\underline{\boldsymbol{s}}$ and $\underline{\boldsymbol{t}}$ be the first steps in $\Gamma$ and $\Delta$ which makes Rank $(\Gamma[-\underline{\boldsymbol{s}}])$ and Rank $(\Delta[\boldsymbol{-} \mathbf{t}])$ positive, respectively. Then, the number of steps of $\Delta[\underline{t-}$ is less than that of $\Gamma[s-]$.

Accordingly, after a finite number of modulation steps, we would arrive at a proof-note with no B-steps and no C-steps.

\section{REFERENCES}

Gentzen, G. [1] Untersuchungen über das logische Schliessen, Math. Z. 39 (1934), 176-210, 405-431.

ONo, K. [1] On a practical way of describing formal deductions, Nagoya Math. J., 21 (1962), $115-121$.

[2] A certain kind of formal theories, Nagoya Math. J., 25 (1965), 59-86.

[3] On universal character of the primitive logic, Nagoya Math. J., 27-1, (1966), 331-353.

[4] Reduction of logics to the primitive logic, J. Math. Soc. Jap., Vol. 19, No. 3 (1967), 384-398.

Mathematical Institute, Nagoya University. 